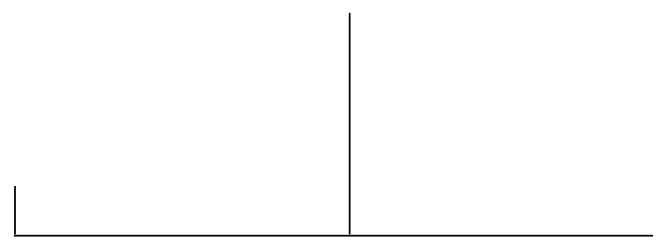

Rev. Latinoam. Psicopat. Fund., São Paulo, v. 15, n. 2, p. 240-250, junho 2012

\title{
Le symptôme dans la psychothérapie d'un enfant dysphasique. Analyse de sa fonction dynamique dans une perspective psychanalytique
}

Julio C. Guillén

Catherine Dupuis-Gauthier

Dans cet article, à partir d'un cas clinique, nous analysons la spécificité de la prise en charge psychothérapeutique de l'enfant dysphasique. Notre objectif principal est de montrer comment la succession de moments logiques donne une place à chaque fois nouvelle au symptôme. L'étude du déroulement de la séquence clinique, permet d'interroger la valeur du symptôme et son lien indissociable à la parole, dans la relation transférentielle, pour rendre compte du processus de subjectivation à l'œuvre chez cet enfant. En outre, ce travail amène à préciser la particularité de la démarche psychothérapeutique par rapport à celles des autres professionnels intervenant auprès des enfants dysphasiques.

Mots clés: Dysphasie, dynamique, psychanalyse, langage 


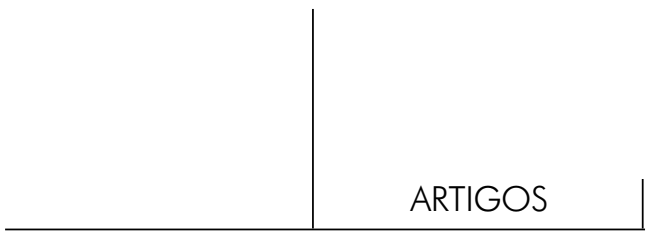

Introduction

Dans cet article, nous proposons de réfléchir, dans une perspective psychodynamique, à la fonction du symptôme comme support d'élaboration psychique, dans le cadre de la psychothérapie d'un enfant présentant une dysphasie (Rapin et Allen, 1983, 1988; Gérard, 1991) plus fréquemment appelée aujourd'hui trouble spécifique du langage oral (Uzè, 2000). Actuellement, dans le champ des troubles instrumentaux, l'évolution des connaissances conduit à une approche compréhensive multifactorielle permettant une lecture dans une triple perspective épistémologique, à savoir, neurobiologique, cognitive et psychodynamique (Dupuis, C. 2000). Chacune de ces approches engendre une interprétation spécifique des symptômes déterminant le type d'intervention: rééducation du langage, remédiation cognitive ou psychothérapie. Or, nous pensons que la pertinence des différentes propositions thérapeutiques dépend du niveau de développement global de l'enfant et de la place que le trouble occupe dans son fonctionnement psychique à un moment donné (Dupuis et al., 2006). L'âge, le niveau de développement sensori-moteur et cognitif, les aptitudes relationnelles et la demande des parents, conditionneront ainsi les possibilités mais aussi la pertinence de l'intervention.

Dans le cadre spécifique des dysphasies de développement, il est nécessaire de distinguer, d'une part, le symptôme comme trouble instrumental, dans le sens d'une impossibilité pour l'enfant d'actualiser la potentialité de la fonction langagière et, d'autre part, le symptôme comme nouage complexe et constructif pouvant faire l'objet d'un travail psychique de "subjectivation" (Cahn, 1991). Toutefois, ces deux aspects du symptôme s'expriment toujours d'une façon plus ou moins entremêlée rendant difficile l'évaluation et aussi la décision thérapeutique. En effet, la rencontre avec un enfant présentant des troubles spécifiques de l'organisation du langage pose la question de la distinction et de l'articulation des différents enjeux du symptôme, déterminés par la représentation que chaque professionnel peut en avoir en fonction de son ancrage théorique. Plus précisément, même si chaque type d'intervention a une place et une fonction pertinente par rapport à l'évolution de l'enfant, l'approche psychothérapique est spécifique. Celle-ci consiste à favoriser l'ouverture d'un potentiel de créativité, impliquant nécessairement la notion 
de conflit psychique inhérente à tout processus de développement qui respecte la position de l'enfant en tant que désirant.

Tout au long de ce processus, le psychothérapeute, tout comme l'enfant, auront à faire aux idéaux des parents, de l'école et de la société avec lesquels il leur faudra composer. Ainsi, même si l'objectif commun à toute prise en charge est d'offrir la possibilité d'acquérir et de développer de nouvelles compétences, chacune de ces approches va privilégier une voie et une technique spécifiques. Ce qui caractérise l'axe psychothérapique est l'attention portée à la place singulière de l'enfant et à la signification subjective du symptôme dans le transfert et ceci au-delà, éventuellement, de toute demande normative de l'entourage. L'attention portée à cette subjectivité est le préalable indispensable à l'avènement du sujet dans le processus même d'acquisition.

À partir du cas clinique d'un enfant présentant un trouble spécifique du langage oral, nous étudierons comment un symptôme phobique, s'installant à un moment précis, favorise l'émergence d'une demande de psychothérapie individuelle dans le cadre d'une prise en charge institutionnelle déjà en place. Au décours de ce suivi, nous assisterons à la transformation du symptôme à la faveur des enjeux transférentiels constituant le point de départ et le noyau même du travail psychothérapique. Le changement de position subjective ainsi induit permettra, chez cet enfant, le développement d'une dynamique psychique plus souple et plus complexe, se reflétant notamment dans l'amélioration de la qualité de son langage.

\section{Presentation et analyse du cas}

C'est en raison d'un passé marqué par des difficultés spécifiques d'organisation du langage ayant engendré un diagnostic tardif de dysphasie, que Kevin est orienté dans un centre spécialisé dans le traitement de ce type de troubles à l'âge de 8 ans.

De son histoire scolaire, nous savons qu'il intègre l'école maternelle à l'âge de trois ans et que, dès la moyenne section, ses parents sont informés par l'institutrice de difficultés de langage et de problèmes relationnels. En effet, il est décrit comme un enfant solitaire, très inhibé dans l'échange avec ses pairs, ceci entravant à la fois ses capacités d'intégration sociale et d'acquisitions scolaires. A l'issue de la grande section de maternelle, la proposition de redoublement faite aux parents sera refusée, ceux-ci préférant changer leur enfant d'école pour lui permettre de passer dans la classe de niveau supérieur. Cette décision marquera le début d'un parcours scolaire fait de nombreuses ruptures puisque Kevin changera encore deux fois d'établissement scolaire. Après deux années de grande 


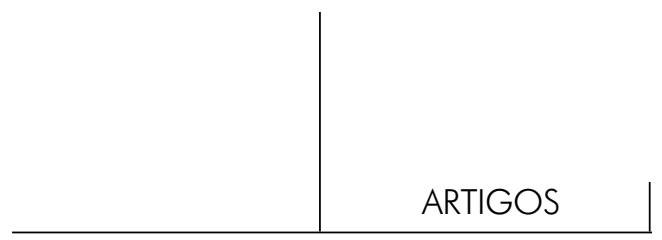

section de maternelle, il sera maintenu en CP ordinaire avant d'intègrer une CLISS à l'âge de 8 ans.

C'est à ce moment que les parents de Kévin décident de rencontrer pour la première fois une orthophoniste qui engage immédiatement une rééducation. Après une année de travail laborieux, elle leur suggérera de consulter dans un centre de diagnostique des troubles du langage, notamment en raison des difficultés d'acquisition de la lecture. Ces consultations aboutiront, après plusieurs mois d'investigation et de bilans neuropédiatrique, psychométrique et orthophonique, au diagnostic de dysphasie de développement de type expressif et à l'orientation vers un établissement spécialisé où Kevin entrera à l'âge de 10 ans.

Cette orientation constitue une césure dans la vie de cet enfant en raison de l'éloignement géographique de l'institution d'avec le domicile mais aussi de la rupture familial qu'impose le passage d'un régime d'externat à un semi-internat. En effet, jusqu'alors inscrit dans une école primaire proche de son domicile, la mère de Kevin le conduisait matin, midi et soir à l'école et déjeunait tous les jours avec lui. Dorénavant, il devra prendre le taxi le matin et le soir, pour parcourir une longue route l'amenant à l'institution où il passera la journée complète, tous les jours de la semaine. C'est précisément à partir de cet éloignement que s'organiseront progressivement les symptômes phobiques, signalés par la mère quatre mois après l'arrivée de Kevin au centre. C'est ce symptôme qui sera à l'origine de la demande de psychothérapie pour cet enfant.

$\mathrm{Au}$ cours du premier entretien, la présentation de Kevin frappe par son aspect soigné et policé, son attitude est réservée, voire inhibée. Assis sur une chaise face au bureau, l'échange est assez difficile, Kevin est immobile, il parait craintif et manque de spontanéité. L'attitude corporelle est crispée, Kevin est visiblement dans l'attente de l'intervention du thérapeute, qu'il semble incapable de solliciter directement et verbalement, la communication non-verbale, elle aussi, est très pauvre. Le regard est franc et il accroche l'interlocuteur. Le visage peu expressif, presque sombre révèle une difficulté à s'engager dans la relation.

Au cours de l'entretien suivant, Kevin commencera à exprimer les raisons de ses difficultés à sortir de chez lui ou de l'institution en disant: "peur de me perdre", "peur d'être oublié" (sic). On apprend également que ses conduites phobiques sont apparues dans le cadre des sorties en groupe organisées par l'institution. Il se souvient en particulier qu'à l'occasion d'une promenade en forêt, alors qu'il s'était isolé à l'arrière du groupe, voyant ses camarades s'éloigner, il s'est senti effrayé. On peut supposer alors un vécu d'angoisse intense à l'idée d'être oublié, abandonné.

Progressivement, dans le cadre du suivi psychothérapique, d'autres éléments confirmeront les difficultés de séparation de Kevin, tout particulièrement lorsqu'il évoque ses troubles du sommeil. Ceux-ci s'organisent autour de son incapacité 
à se coucher avant d'avoir parlé à sa mère, il n'arrive pas à s'endormir quand il est seul. Il dit: "bouge tout le temps y'a mon frère dans mon lit ... dors avec mon frère, des fois avec ma mère ... peur d'étouffer" (sic). Ces craintes vont s'installer et envahir son existence, la peur de l'étouffement notamment pourra être mise en lien avec les laryngites aigues dont il a souffert dès le plus jeune âge et qui faisaient craindre à la mère que son "bébé ne puisse plus respirer". Ces inquiètudes massives, pesant de tout leur poids sur les relations précoces entre la mère et l'enfant, pourraient être mises en lien avec des angoisses agonistiques précoces telles que Winnicott (1972) pouvait les décrire.

Par la suite, Kevin évoquera un ancien cauchemar qui viendra constituer un noeud structurel lui permettant un début de subjectivation et d'historicisation des troubles tout en organisant une nouvelle configuration transférentielle: en haut d'une butte, frère et copains avec moi, en haut ... tout en bas des crocodiles... de l'eau. Les copains poussé en bas, est tombé ... les crocodiles m'ont pas mangé. (sic)

Ce moment crucial dans le travail psychothérapique aura des répercutions au moins à trois niveaux:

1. pour Kevin, en tant qu'il favorise le développement de ses capacités de symbolisation;

2. pour l'équipe, en tant qu'il offre une possibilité de représentation et une signification aux conduites jusqu'alors énigmatiques de l'enfant;

3. pour le thérapeute, en tant qu'il est reconnu par le patient dans cette position subjectivante, instaurant une relation spécifique et différente de celles que Kevin entretient avec les membres sa famille et les autres professionnels. L'interprétation des angoisses abandonniques et persécutives devenant possible dans le transfert à partir du matériel du rêve.

En effet, c'est à travers la narration et l'actualisation de ce cauchemar que le psychothérapeute est inscrit, par Kevin, dans une nouvelle place et une nouvelle fonction qui sollicitent directement ses capacités de rêverie, de contenance et de transformation (cf. Bion, 1979).

La position de l'objet, telle que perçue par l'enfant, caractérisée jusqu'alors par l'alternance entre une présence envahissante et une absence absolue, empêche l'installation de la "bonne distance" nécessaire à la construction d'un espace symbolique partagé, condition de possibilité pour la constitution d'une "matrice active de signification" (Penot, 2004) à partir de laquelle les signifiants à venir trouveront une place. Ainsi, les angoisses abandonniques peuvent être évoquées dans un énoncé, certes élémentaire, mais qui s'adresse maintenant au psychothérapeute. "Tomber", "être dévoré" peuvent, à partir de ce moment, se dire, témoignant ainsi d'un début d'élaboration, dans le contexte d'une relation suffisamment contenante mais non intrusive (Houzel, 1987). Cette nouvelle mise en forme du symptôme dans une parole explicitement adressée nous éloigne ainsi 


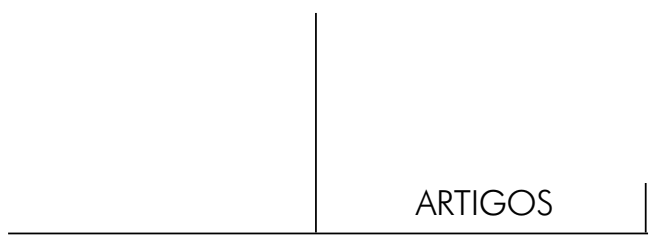

du comportement phobique dans sa dimension "agie", non médiatisé par la parole. On remarque que cet aménagement de la relation en termes de distance symbolique a un effet presque littéral chez Kevin qui accepte maintenant d'aller à la piscine, à condition de pouvoir rester à proximité de son enseignant.

Après plusieurs mois de travail, ce sont les symptômes de type obsessionnel qui apparaîtront sous la forme d'un besoin exagéré de contrôle: lavage de mains, lenteur psychomotrice, bizarrerie des conduites alimentaires ayant entraîné une perte de poids et un ralentissement généralisé. Kevin mange très lentement et très peu car il trie son alimentation par peur d'ingurgiter des produits potentiellement "dangereux". Cette organisation symptomatique marque un second temps essentiel dans le processus de subjectivation de Kevin - processus au sens d'une continuité supportant la faille où pourra s'inscrire le sujet comme émergeant, dans le sens de "ce qui se creuse dans l'expérience de la parole" (Lacan, 1953).

A partir de l'apparition dans son discours de ces craintes nouvelles d' "être empoisonné" signant la présence d'angoisses persécutives, c'est la fonction de l' Autre qui s'en trouve changée.

Il s'agit maintenant d'une relation caractérisée par la confrontation et qui fait apparaître l'opposition persécuteur-persécuté soulevant en même temps le problème de l'agressivité et de la mort. La lenteur du comportement compromet les activités quotidiennes de la mère débordée par la manière de procéder de son fils qui la force, soit à patienter, soit à lui répéter sans cesse de se dépêcher.

C'est donc la demande de l'Autre qui entre en scène à ce moment: "mange", "dépêches-toi", "sors de la douche". Ainsi, Kevin peut, par cette nouvelle symptomatologie qui "l'oblige" à tout ralentir, tenir l'autre en souffrance, refuser sa demande et mettre en question ce qu'on lui offre. Manger ne fait pas nécessairement du bien.

Le thème de la mort apparaît, d'une part, dans l'explication qu'il donne de ses comportements alimentaires: "enlève noir dans mon assiette, peur de la drogue, du poison", "la drogue ça fait devenir tout maigre, on ne sent plus rien" (sic). D'autre part c'est à travers le contenu de son récit que se révèlent les tendances agressives: "Mon chien il est mort, la tortue, le poisson rouge, les oiseaux ... le père de ma mère, le petit bébé de ma tante ... Il est sorti du ventre ... mort comme ça, il était trop petit" (sic).

L'accession à une meilleure capacité d'expression associée à une certaine forme d'opposition dans la relation fait suite à l'attitude jusqu'alors très soumise de Kevin. En effet, il peut verbaliser le contenu de certaines des angoisses qui l'habitent. Dorénavant, c'est toute l'ambivalence pulsionnelle qui peut se dire à travers la crainte d'introjecter de mauvais objets (peur d'être empoisonné) ou que les bons objets intériorisés soient attaqués (bébé mort). L'expulsion au dehors de soi est synonyme de la perte, voire de l'anéantissement de ces objets internes qu'il 
faut absolument préserver en les maintenant au dedans de soi. Ce mécanisme est un des enjeux fondamentaux de la parole, puisque parler implique un renoncement pulsionnel. Chez l'enfant dysphasique celui-ci se traduit notamment par la difficulté à se séparer de l'objet "voix" (Bergès, 1994).

Evidemment, chez Kevin il s'agit d'un refus de la demande de l'Autre qui ne se réduit pas au comportement mais qui apparaît aussi à travers l'insistance au niveau de la parole de l'expression: “c'est tout”, qu'il utilise régulièrement pour ponctuer ses propos. De cette façon, il essaie non seulement d'interdire à l'interlocuteur toute possibilité de continuer le dialogue, mais aussi d'éviter tout risque d'extérioriser des objets internes voués à une destruction certaine. C'est du moins ce que nous permet d'inférer l'analyse des éléments du transfert.

A partir de ce moment, certains comportements, activités, narrations de Kevin commencent à installer une problématique oedipienne qui fait appel à la fonction du père et, bien sûr, à l'objet du désir de sa mère. Ceci s'exprime d'abord par un cauchemar "positif":

y a mon petit frère et ma famille, moi faisais un câlin à mon frère, mon père mis un crocodile près de moi, après mangé... le pont de bois on est tous tombé et mon père rattrapé toute la famille ( $\mathrm{sic}$ ).

Puis par l'évocation du travail de son père et de son absence certaines nuits: [tous les] jeudis quand mon père travaille nuit, dormir à trois [Kevin et son frère avec leur mère] et mon père y revient demain, papa y dort pas et après-midi repose ( $\mathrm{sic}$ ). On perçoit la fonction que Kevin tente d'occuper auprès de la mère lorsque le père est absent, et son souhait inconscient de le remplacer, bien qu'il éprouve pour lui des sentiments ambivalents d'amour et de haine.

L'apparition de la problématique oedipienne constituera une nouvelle ouverture pour la conduite du traitement psychanalytique à venir.

En effet, considérant que "être subjectivé, c'est prendre place dans un sujet comme valable pour un autre sujet" (Lacan, 1960, p. 285), l'enjeu ne se réduit pas à assurer un "bonne distance" entre le sujet et l'objet qui est toujours singulière et sans commune mesure avec les autres. Certainement, la construction d'une "bonne distance" est la tache préalable à tout aménagement d'un espace thérapeutique en lien avec un possible espace de représentation psychique. Cependant, la dynamique œdipienne viendra installer une complexité nouvelle dans une dynamique de subjectivation à quatre éléments: l'enfant et les deux figures parentales associées à "ce qui est valable" dans le triple sens d'appréciable - valeur phallique -, acceptable - accepté par l'autorité - et vraisemblable - qui a un fondement. A présent, ceci constitue encore une zone à déployer dans le traitement de Kevin.

En outre, les effets de ce travail seront relayés par l'orthophoniste qui nous fera part des progrès langagiers de Kevin au niveau de l'expression verbale, se rapprochant de celui de son groupe d'âge. 


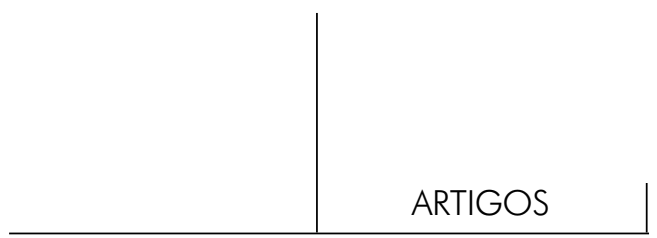

Conclusion

Dans cet article nous avons voulu insister sur la dimension dynamique du symptôme à travers l'analyse d'un cas clinique caractérisé par la succession de quatre moments décisifs de la construction symptomatique:

1. L'apparition du symptôme phobique dans le plan comportemental qui provoque la demande de consultation.

2. Le nouage transférentiel de ce symptôme à partir de la narration d'un premier cauchemar et de l'expression verbale de ses angoisses. Une demande à l'autre apparaît alors pour "tenir en vie". Il s'agit de l'ébauche d'un espace symbolique, même s'il est encore très fragile comme le suggère la présence de l'angoisse de dévoration.

3. L'apparition du symptôme obsessionnel qui s'accompagne du changement des angoisses exprimées en termes de persécuteur/persécuté, transforme la place de Kevin et celle du thérapeute dans la relation. C'est la demande de l'autre qui est ici en jeu.

4. L'émergence dans le discours de la problématique oedipienne constituera un nouveau virage qui permettra au thérapeute de "faire jouer" dans le transfert la dimension de la castration.

En conclusion, ces quatre temps nous montrent qu'au-delà du contenu imaginaire des angoisses exprimées par Kevin, l'analyse de la dynamique du symptôme doit tenir compte de l'aspect logique des changements successifs de sa position vis-à vis de l'Autre tout au long du traitement. Il s'agit de “temps logiques [qui] ne peuvent se dérouler que dans une certaine succession" (Lacan 1957, p. 198).

Enfin, la particularité de notre démarche, qui permet de conduire progressivement un enfant dysphasique dans le décours d'un traitement psychanalytique, s'avèrera dans la pratique positivement articulé à d'autres types de soins, confirmant ainsi la pertinence et l'efficacité d'une approche pluridisciplinaire qui respecte la spécificité technique et épistémologique de chaque mode d'intervention.

\section{References}

Bergès, J.; BALBo, G. La dysphasie, le leurre: techniques en psychanalyse. In: L'enfant et la psychanalyse. Paris: Masson, 1994. p. 153-166.

BIon, W.R. Aux sources de l'expérience. Paris: PUF, 1979.

CAHN, R. Du sujet. Revue française de psychanalyse, v. 55, n. 6, p. 1354-1490, 1991. 
Dupuis, C. Pour une conception multifactorielle des troubles sévères de développement du langage. Orthomagazine, n. 34, p. 8-11, 2000.

Dupuis-Gauthier, C.; Guillén, J. et Beaune, D. L'enfant dysphasique: un sujet en situation clinique. A propos de la prise en charge psychothérapeutique. Neuropsychiatrie de l'enfance et de l'adolescence, n. 54, p. 396-400, 2006.

GÉRARD, C.L. L'enfant dysphasique. Paris: PUF, 1991.

Houzel, D. Le concept d'enveloppe psychique. In: Anzieu et al. Les enveloppes psychiques. Paris: Dunod, 1987. p. 23-45.

LaCAN, J. (1953). Le séminaire. Livre I. Les écrits techniques de Freud. Paris: Seuil, 1975.

1998.

. (1957). Le séminaire. Livre V. Les formations de l'inconscient. Paris: Seuil, . (1960). Le séminaire. Livre VIII. Le transfert. Paris: Seuil, 2001.

Penot, B. Travailler psychanalytiquement à plusieurs : la reprise d'un temps premier du processus subjectivant. Adolescence, v. 50, n. 4, p. 833-842, 2004.

Rapin, I.; Allen, D.A. Developmental language disorders: nosologic consideration. In: KIRK, U. (Eds.). Neuropsychology of language, reading ans spelling. New York: New York Academic Press, 1983. p. 155-184.

Rapin, I.; Allen, D.A. Syndromes in developmental dysphasia and adult aphasia. In: Plum, F. Language, communication and the brain. New York: Raven Press, 1988. p. 57-75.

.;____. Syndromes in developmental dysphasia and adult aphasia. In: Plum, F. Language, communication and the brain. New York: Raven Press, 1988. p. 57-75.

Uzé, J. Dysphasie. In: Dictionnaire de psychopathologie de l'enfant et de l'adolescent. Sous la direction de D. Houzel, M. Emmanuelli et F. Gerstle-Moggio. Paris: PUF, 2000.

Winnicott, D.W. (1972). La crainte de l'effondrement. In: La crainte de l'effondrement et autres situations cliniques. Paris: Gallimard, 2000.

\section{Resumes}

(O sintoma na psicoterapia de uma criança disfásica. Análise da sua função dinâmica a partir de uma perspectiva psicanalítica)

Neste artigo analisamos a especificidade da cobertura psicoterapêtica de crianças com disfasia a partir de um caso clínico. Trata-se aqui de mostrar a sucessão 
de momentos lógicos que dão um lugar cada vez novo aos sintomas. A seqüência clínica vai permitir-nos a questionar o valor do sintoma e sua ligação inseparável com a palavra na relação de transferência para refletir o processo de historicização da subjetividade da criança. Além disso, este percurso vai permitir-nos situar a especificidade da abordagem psicoterapêutico comparados aos de outros profissionais que trabalham com crianças com disfagia.

Palavras-chave: Disfasia, dinâmica, psicanálise, linguagem

(The symptom in the psychotherapy of a dysphasic child. Analysis of his dynamic function from a psychoanalytical perspective)

In this article we analyze the specificity of the psychotherapy of a dysphasic child, based on a clinical case. Our aim is to show the succession of logical moments that give ever newer places to the symptom. The study of a particular clinical sequence will allow us to question the value of the symptom and its inseparable association with speech in the transference relationship, in order to discuss the "subjectivation" process at work in this child. This discussion will also allow us to situate the main specific characteristics of the psychotherapeutic approach as compared to approaches of other professionals who treat dysphasic children.

Key words: Dysphasia, dynamics, psychoanalysis, language

(El síntoma en la psicoterapia de un niño disfásico. Análisis de su función dinámica desde una perspectiva psicoanalítica)

En este artículo, a partir de un caso clínico, analizaremos la especificidad del tratamiento psicoterapéutico de un niño disfásico. Nuestro objetivo principal es mostrar como la sucesión de momentos lógicos confiere en cada momento una nueva posición al síntoma. El estudio del desarrollo de la secuencia clínica, permite interrogar el valor del síntoma y su relación indisociable con la palabra, en la relación transferencial, lo que permite reflexionar sobre el proceso de historización de la subjetividad del niño en el caso considerado. Además, este recorrido nos permitirá precisar la particularidad del trabajo psicoterapéutico respecto al de otros profesionales que intervienen en el tratamiento de niños con disfasia.

Palabras clave: Disfasia, dinámica, psicoanálisis, lenguaje

(Das Symptom in der Psychotherapie eines dysphasischen Kindes. Analyse seiner dynamischen Funktion aus psychoanalytischer Perspektive)

Ausgehend von einem klinischen Fall, wird die Spezifik der psychotherapeutischen Betreuung von Kindern mit Dysphasie untersucht. Es geht darum, die Reihenfolge logischer Momente aufzuzeigen, die den Symptomen eine ständig neue Bezugsgröße geben. Die klinische Sequenz wird die Hinterfragung des Stellenwertes des Symptoms und seine untrennbare Verbindung mit dem Wort in der 
Übertragungsbeziehung erlauben, um den Historisierungsprozess der Subjektivität des Kindes zu reflektieren. Außerdem erlaubt dieser Vorgang, die Spezifik des psychotherapeutischen Ansatzes im Vergleich zu anderen Spezialisten, die mit dysphasischen Kindern arbeiten, zu situieren.

Schlüsselwörter: Dysphasie, Dynamik, Psychoanalyse, Sprache

Citação/Citation: Guillén, J.C.; Dupuis-Gauthier, C. Le symptôme dans la psychothérapie d'un enfant dysphasique. Analyse de sa fonction dynamique dans une perspective psychanalytique. Revista Latinoamericana de Psicopatologia Fundamental, São Paulo, v. 15, n. 2, p. 240-250, jun.2012.

Editor do artigo/Editor: Prof. Dr. Manoel Tosta Berlinck

Recebido/Received: 28.2.2011 / 2.28.2011 Aceito/Accepted: 13.5.2011 / 5.13.2011

Copyright: () 2009 Associação Universitária de Pesquisa em Psicopatologia Fundamental/ University Association for Research in Fundamental Psychopathology. Este é um artigo de livre acesso, que permite uso irrestrito, distribuição e reprodução em qualquer meio, desde que o autor e a fonte sejam citados / This is an open-access article, which permits unrestricted use, distribution, and reproduction in any medium, provided the original author and source are credited.

Financiamento/Funding: Os autores declaram não ter sido financiados ou apoiados / The authors have no support or funding to report.

Conflito de interesses/Conflict of interest: Os autores declaram que não há conflito de interesses / The authors declares that has no conflict of interest.

Julio C. Guillén

Docteur en Psychologie.

Laboratoire SHS-CEC - Unité de Recherche en Psychologie OCeS (Organisation, Clinique et Sujet). Université Catholique de Lille. Faculté Libre des Lettres et sciences Humaines.

60 Bd Vauban BP 109. F-59016 Lille Cedex

EPSM Agglomération Lilloise - CMP Franco Basaglia]239, Rue du Faubourg de Roubaix 59800 Lille, France

e-mail: julio.guillen@icl-lille.fr

Catherine Dupuis-GauthiER

Docteur en Psychologie.

MCF Psychologie clinique et pathologique, Université de Lille 3

Rue du barreau

BP 60149, 59653 Villeneuve d'ascq cedex

CRPMS (EA 3522) Université Paris Diderot

Service de pédopsychiatrie, CHRU de Lille

e-mail: catherine.dupuis@univ-lille3.fr

Rev. Latinoam. Psicopat. Fund., São Paulo, v. 15, n. 2, p. 240-250, junho 2012 